\title{
A study on outdoor to indoor penetration path loss at 2 and $5 \mathrm{GHz}$
}

\author{
Yuta Mizuno ${ }^{\text {a) }}$, Kentaro Nishimori, and Ryotaro Taniguchi \\ Graduate School of Science and Technology, Niigata University, \\ 8050 Ikarashi 2-no-cho, Nishi-ku, Niigata 950-2181, Japan \\ a)mizuno@gis.ie.niigata-u.ac.jp
}

\begin{abstract}
The 5-th generation of mobile communication systems $(5 \mathrm{G})$ are being actively investigated all over the world. Various systems exist when considering the microwave bands after the introduction of the $5 \mathrm{G}$ systems. It is very important to consider the microwave propagation characteristics in order to minimize possible interferences among systems. In this paper, we propose a simple outdoor to indoor $(\mathrm{O} 2 \mathrm{I})$ propagation model based on the 2 and 5-GHz bands. The proposed model focuses on the variation in incident angle on a vertical plane when the microwaves move from O2I environments. This model shows realizes the additional loss by using only angles on the vertical plane.
\end{abstract}

Keywords: radio wave propagation, penetration loss, loss factor Classification: Antennas and Propagation

\section{References}

[1] H. Papadopoulos, C. Wang, O. Bursalioglu, X. Hou, and Y. Kishiyama, "Massive MIMO technologies and challenges towards 5G," IEICE Trans. Commun., vol. E99-B, no. 3, pp. 602-621, Mar. 2016. DOI:10.1587/transcom. 2015EBI0002

[2] H. Kimoto, K. Nishimori, N. Omaki, K. Kitao, and T. Imai, "Experimental evaluation on outdoor to indoor propagation characteristics for multiple microwave bands," IEICE Commun. Express, vol. 5, no. 3, pp. 85-89, Mar. 2016. DOI:10.1587/comex.2015XBL0183

[3] 3GPP TR 36.873, v.1.1.1, "3rd Generation Partnership Project; Technical Specification Group Radio Access Network; Study on 3D channel model for LTE," 2013-09.

\section{Introduction}

The 5-th generation of mobile communication systems (5G) are being actively investigated all over the world. Although 5G systems mainly employ millimeter waves, they also make use of frequency bands $(<6 \mathrm{GHz})$ [1]. Various systems have been proposed for the use of microwave bands in $5 \mathrm{G}$ systems, especially for frequencies of $900 \mathrm{MHz}, 2.4 \mathrm{GHz}$, and $5 \mathrm{GHz}$. 
Here, we focused on the outdoor to indoor (O2I) propagation path loss, since terminal stations are used both in outdoor and indoor environments, while base stations are installed only in outdoor environments. Other O2I models have been previously proposed in [2, 3]. A fixed value was used in [2]; however, the model in [3] considered the incident angles on the horizontal plane and used calculated the O2I factors (path loss coefficients) for frequencies of 0.8, 2.2, and $4.7 \mathrm{GHz}$.

In this paper, we propose a simple O2I propagation model based on the 2.4 and 5.1-GHz bands. These bands are of particular interest, since they are used by $5 \mathrm{G}$ $(5.1 \mathrm{GHz})$ and other wireless systems (e.g., WLAN).

The proposed model focuses on the variation in incident angle on a vertical plane when the microwaves move from an O2I environments. It shows that the received power is greatly influenced by the incident angles on the vertical plane. Another simple model was employed to analyze the O2I factor, showing that the difference between the real and the calculated path loss is very small.

The rest of the manuscript is structured as follows: Sect. 2 shows the results of the environmental measurements, while Sect. 3 presents a simple model that can be used to calculate the additional path loss indoors, based on the incident angle on a vertical plane.

\section{Measurement of $\mathrm{O} 2 \mathrm{I}$ penetration loss}

\subsection{Measurement environment}

We measured the O2I penetration path loss between two buildings at Niigata University, considering frequencies of 2.425 and $5.12 \mathrm{GHz}$. The incident angle $\theta$ was defined as the angle comprised between the straight line connecting the receiving side to the $0 \mathrm{~m}$ point on the transmitting side, and another line parallel to the ground. The incident angles comprised between the receiving (at the $1 \mathrm{st}$ floor) and transmitting (at the 1st to 8th floors) sides were of $4.52^{\circ}, 1.39^{\circ}, 1.73^{\circ}$, $4.86^{\circ}, 7.96^{\circ}, 11.01^{\circ}, 14.01^{\circ}$, and $16.92^{\circ}$. The incident angles comprised between the receiving (at the 7 th floor) and transmitting (at the 1 st to 8 th floors) sides were instead of $23.20^{\circ}, 20.50^{\circ}, 17.69^{\circ}, 14.80^{\circ}, 11.83^{\circ}, 8.79^{\circ}, 5.71^{\circ}$, and $2.58^{\circ}$. We compared the received power with the transmit distance. Fig. 1 shows the results of the measurement environment: apparently, a continuous wave (CW) was transmitted from the 1st floor to the 8th floor in the right building while a transmitter was moved along a corridor. The transmit distance was of $26 \mathrm{~m}$; moreover, the receivers were located at the 1 st and 7 th floors of the receiving building.

\subsection{Measurement result}

The measured received power was corrected for the loss due to free space $(\alpha=2)$, to assure that this variable would not affect our results. The normalized received power was standardized so that the maximum received power of the data obtained at the transmitters 1 st to 8 th floors was $0 \mathrm{~dB}$ for the received power. Moreover, the additional loss inside the building was calculated from the normalized received power. Fig. 2 shows the additional loss for transmitters at 2nd, 4th, 6th and 8th floors and the receivers at the 1st and 7th floors, considering frequencies of 2.425 and $5.12 \mathrm{GHz}$. The correspondent incident angles were large and the additional loss 


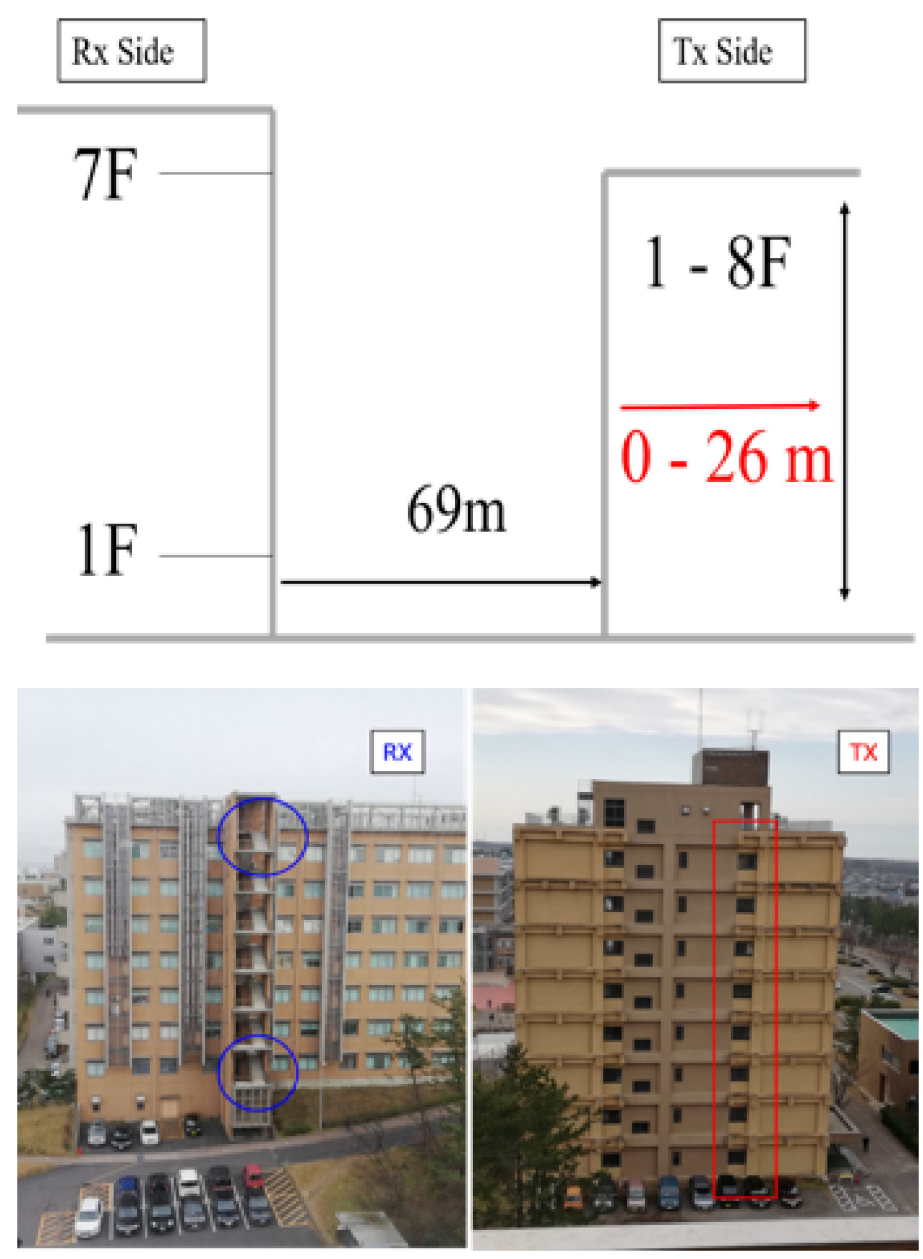

Fig. 1. Measurement environment

tend to increase, regardless of the receivers located at the 1st and 7th floors. The incident angles between transmitter at 2 nd and the 8 th floors and the receivers at the 1 st (and 7th floors) were of $1.39^{\circ}\left(20.50^{\circ}\right)$ and $16.92^{\circ}\left(2.58^{\circ}\right)$ : these angles on the vertical plane determined the indoor penetration path loss.

\section{Analysis of measurement results}

The O2I factor $\gamma$ is a variable representing the magnitude of the increase in additional loss that occurs with increasing distance inside the building [3]. Assuming an additional loss of $L \mathrm{~dB}$, the $\mathrm{O} 2 \mathrm{I}$ factor and the transmit distance should be equal to $\gamma$ and $d \mathrm{~m}$, respectively. The common logarithm regression curve of Eq. (1) was approximated using the least-squares method.

$$
\begin{aligned}
& L=\gamma \log _{10}(d+1)+\beta \quad[\mathrm{dB}] \\
& \gamma=\frac{\sum_{i=1}^{N} L_{i}}{\sum_{i=1}^{N}\left(\log _{10}\left(d_{i}+1\right)\right)} \\
& \gamma=a \theta+b
\end{aligned}
$$

In Eq. (2), $N, L_{i}$, and $d_{i}$ denote the number of data samples, $i$-th additional loss and $i$-th distance for $L_{i}$, respectively. 

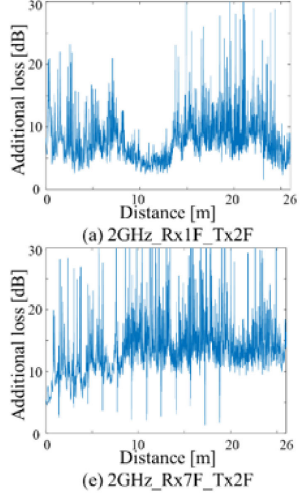

(e) $2 \mathrm{GH}$
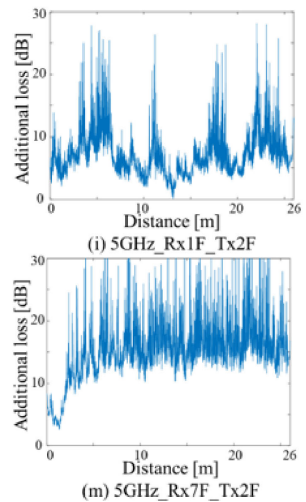

(m) $5 \mathrm{GHz}_{-} \mathrm{Rx} 7 \mathrm{~F}-\mathrm{Tx} 2 \mathrm{~F}$
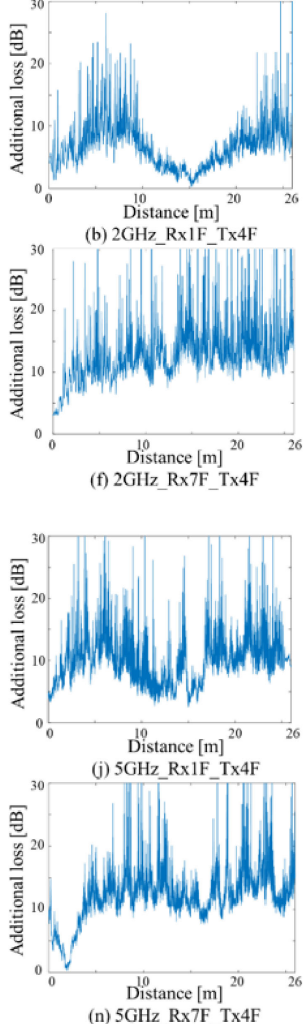
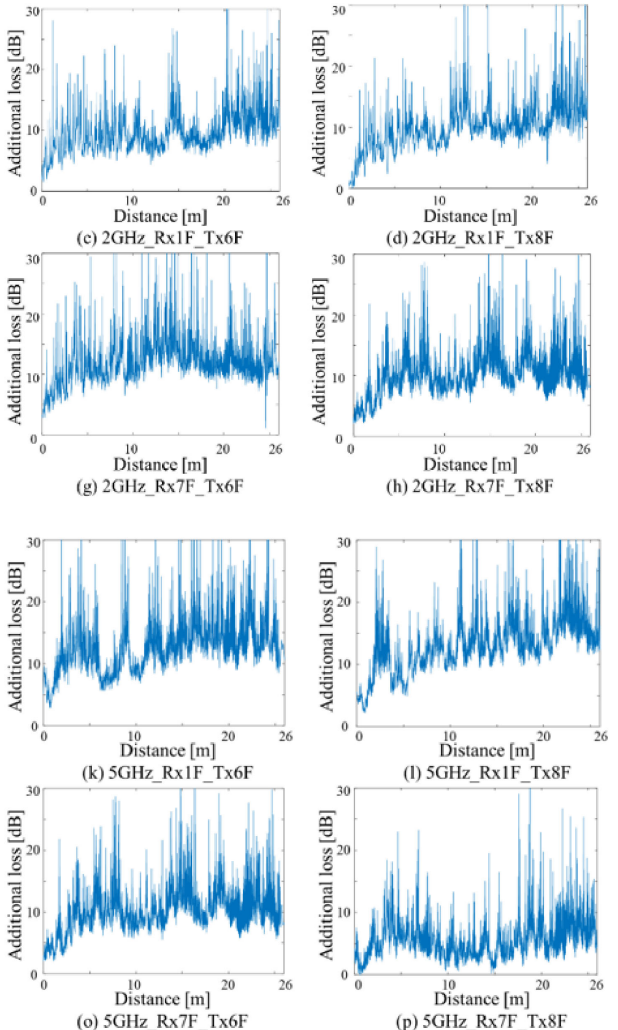

Fig. 2. O2I penetration loss

In Eq. (3), $\theta$ represents the incident angle on the vertical plane, while the value of $\beta$ in Eq. (1) is fixed and determined by frequency. In this analysis, the maximum received power $(0 \mathrm{~dB})$ was taken as the value of $\beta$. The value of $\gamma$ in Eq. (1) was calculated through Eq. (2). The regression line was approximated based on Eq. (3), using the least-squares method on the incident angle $(\theta)$ along the $\mathrm{X}$-axis and the O2I factor $(\gamma)$ along the Y-axis. The results of this analysis are shown in Fig. 3. The trend lines in Figs. 3(a) and 3(b) correspond to Eqs. (4) and (5), respectively, which also describe our approximation of the O2I factor $(\gamma)$.

$$
\begin{aligned}
& \gamma=0.30 \theta+5.94 \\
& \gamma=0.35 \theta+6.39
\end{aligned}
$$

Fig. 3 shows that the O2I factor $(\gamma)$ increased together with the incident angle $(\theta)$, following the trend line. The correlation coefficients between these two

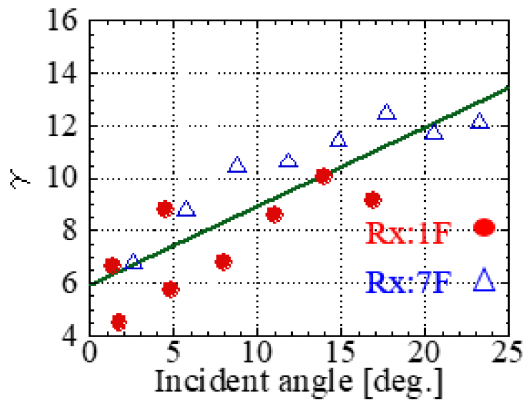

(a) $2 \mathrm{GHz}$

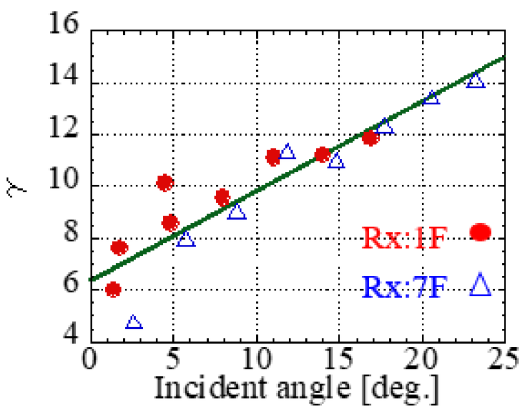

(b) $5 \mathrm{GHz}$ 
variables were equal to 0.84 and 0.92 under frequencies of 2.425 and $5.12 \mathrm{GHz}$. These high correlation coefficient values indicate that the O2I factor $(\gamma)$ can be represented as a linear function of the incident angle $\theta$. The O2I factor should have increased with the incident angle on the vertical plane because, for small incident angles, direct waves can reach even very distant indoor sections. Following this reasoning, both the O2I factor and the incident angle should have been small. In case of high incident angles, direct waves cannot reach indoor sections, and the received waves are reflected and diffracted; therefore, as the incident angles increase, the losses due to reflection and diffraction also tend to increase. The significant attenuation of reflected and diffracted waves suggests that the additional loss with distance and the loss factor also increased. As the incident angle increases, the reflected wave and the diffracted wave are also attenuated significantly, so it is shown that the additional loss with distance increases and the loss factor increases. By comparing Eqs. (4) and (5) we observed that the value of $\gamma$ is larger in Eq. (5): the loss factor increased with the frequency band.

\section{Conclusion}

In this paper, we modeled the O2I path loss considering an incident angle on a vertical plane. We estimated the additional loss by the regression curve of a common logarithm and showed that the calculated O2I factor can be expressed as a linear function of the incident angle.

\section{Acknowledgments}

Part of this study is the result of a research project commissioned by the Ministry of Internal Affairs and Communications ("Research and development of optimal communication method selection technologies corresponding to various radio wave environments in IoT/5G times"). We thank the members of Nishimori laboratory for their help with the measurements and for their precious advice. 